\title{
USE OF LACTIC ACID AND SPAN 80 IN THE FORMULATION OF LIPID BASED IMIQUIMOD VESICLES FOR GENITAL WARTS
}

\author{
SAROJ JAIN ${ }^{*}$, ANUPAMA DIWAN ${ }^{b}$, SATISH SARDANA ${ }^{\mathrm{a}}$ \\ aDepartment of Pharmaceutics, Hindu College of Pharmacy, Sonepat (Hr), India, bDepartment of Pharmaceutical Sciences, Apeejay Stya \\ University, Gurgaon (Hr), India \\ Email: sarojhcp@yahoo.com
}

Received: 27 Oct 2016 Revised and Accepted: 29 Dec 2016

\section{ABSTRACT}

Objective: The objective of present study was formulation development of imiquimod using lactic acid and span 80 for topical delivery to cure genital warts.

Methods: Lipid based vesicles (LBV) of $2 \%$ imiquimod were prepared with phospholipoin 90G, ethanol, lactic acid and span 80 using central composite design. The prepared vesicles were optimized statistically and characterized for particle size, zeta potential, percentage entrapment efficiency (\% EE) and transmission electron microscopy (TEM). The optimized LBV were incorporated into gel formulation which was evaluated and compared with control gel and marketed formulation.

Results: The optimized vesicles had particle size $394.8 \pm 9.6 \mathrm{~nm}$, zeta potential- $16.5 \pm 2.5 \mathrm{mV}, \%$ EE $88.27 \pm 0.45$ and TEM study confirmed the formation of vesicular structure with spherical shape. The gel formulation of imiquimod vesicles showed positive results like spreadability $14.3 \pm 0.34 \mathrm{gcm} / \mathrm{s}$, viscosity $13500 \pm 1.67 \mathrm{cp}$, consistency $6.1 \pm 0.14 \mathrm{~mm}$ and extrudability $16.47 \pm 0.11 \mathrm{~g} / \mathrm{cm}^{2}$. In vitro permeation amount of drug was remarkably lower (10.13\%) than control (87.17\%) and marketed formulation (27.46\%). Results of retained drug for both in vitro as well as in vivo permeation study and local accumulation efficiency (4.021 \pm 0.2292$)$ were considerably higher for LBV gel than control $(0.1008 \pm 0.002513)$ and marketed formulation $(0.8314 \pm 0.0300)$. To understand the mechanism of interaction between skin and vesicles, fourier transform infra-red spectroscopy studies were also done. Results of skin irritancy test and histological examination revealed biocompatible nature of formulation.

Conclusion: Results of in vitro and in vivo studies indicated that this vesicle gel formulation provided efficient and site specific dermal delivery of imiquimod for cure of genital warts.

Keywords: Ethosomes, Genital warts, Human papillomavirus, Imiquimod, Lipid based vesicles, Phospholipid

(c) 2017 The Authors. Published by Innovare Academic Sciences Pvt Ltd. This is an open access article under the CC BY license (http://creativecommons.org/licenses/by/4. 0/) DOI: http://dx.doi.org/10.22159/ijpps.2017v9i2.15915

\section{INTRODUCTION}

Genital warts (GW) are the infection caused by one or more varieties of 100 familiar types of human papillomaviruses (HPVs). They are the visible manifestations and an important issue for women concerned about communication of HPVs and GW to their sexual associates [1]. HPVs types 6 and 11 are cause of most of the GW, cosmetically unacceptable, associated with emotional stress, discomfort and pain [2]. Warts are present in approximately 2-25\% of sexually transmitted disease (STD) patients attending treatment centers in India [3]. Treatments for GW may be applied by the patient, or a physician. Imiquimod $5 \%$ cream therapy applied by the patient straightway enhances immune response to HPV is safe and effective treatment for GW. A reduction in viral number and low recurrence rate is observed following treatment as compared to other treatment options available may be due to stimulation of cell mediated immune response by imiquimod [4].

Imiquimod is a small molecule of imidazoquinoline family, a group of nucleoside analogs that were first synthesized as prospective antiviral agents. At present, it is accepted for external anogenital warts as well as actinic keratosis (AK) and cutaneous lesions transformed into superficial basal cell carcinoma (sBCC) [5].

Growth in medical field along with upward trend for dermatological products has facilitated the materialization of superior topical formulations as drug delivery systems which have many advantages over conventional dosage forms as very easy to use, which make them appealing for patients, avoid first-pass hepatic metabolism, gastric degradation, frequent dosing, and inconvenience of parenterals. These non-invasive drug delivery systems provide sustained therapy with single application as well as offer substitute route of administration and enhanced delivery of active molecule to target site in the body locally [6, 7]. However, at the same time dermal and transdermal delivery of many tiny and big molecules pose significant challenge because of obstinate barrier properties of skin.

The rationale of this research is formulations development of imiquimod for topical delivery to cure GW with low dose therapy than Aldara 5\% imiquimod cream. The formulation of imiquimod presents a peculiar challenge due to its insoluble nature in aqueous solutions at physiological $\mathrm{pH}$. In fact, it has very little solubility in most organic solvents except fatty acids [8]. Particularly, the proposed work is directed to reduce dose strength of imiquimod formulation to deliver an efficacious treatment with enhanced skin targeting for GW with an acceptable safety profile and more convenient for patient use through innovation of novel and improved low dose strength lipid based vesicles (LBV), ethosomes. These ultra-deformable vesicles, composed of phospholipids together with high concentration of ethanol, were developed by Touitou [9]. In the present investigation, lactic acid and span 80 have been used additionally. Central composite design has been employed for optimization of best formulation, further evaluated using different parameters

The formulated LBV have been incorporated in gel formulation which are preferred out of numerous transdermal/topical formulations like creams, ointments, lotions, patches, etc due to ease of application and better precutaneous absorption. Formulations in gel form offer better stability and application property in contrast to cream and ointments because of non-sticky nature and requiring low energy during formulation [10].

The overall objective of this study was to (i) design and characterize LBV of imiquimod for treatment of GW (ii) improve solubility of imiquimod because of its highly hydrophobic nature (iii) optimize various methods 
of increasing drug loading and (iv) evaluate subsequent effects on in vitro and in vivo release and deposition of drug.

\section{MATERIALS AND METHODS}

\section{Chemicals and reagents}

Materials with their source were imiquimod (Glenmark Pharmaceuticals, Mumbai), ethanol (Chansgashu Yanguan Chemicals, China), phospholipoin 90G (LIPOID Company, Germany), span 80 (Thomas Baker (Chemicals) Pvt. Ltd. Mumbai), lactic acid (Central Drug House, Pvt. Ltd. New Delhi) and carbopol 980 NF (Lubrizol). All other reagents were of analytical grade and used without further purification. Double-distilled water was used throughout the study.

\section{Animals}

Animals-Wistar albino rats 6 to $8 \mathrm{w}$ old weighing 120 to $150 \mathrm{~g}$ devoid of any disease was used for study. All investigations were carried out sanctioned by Institutional Ethical Committee (Hindu College of Pharmacy, Sonepat) for the Purpose of Control and Supervision of Experiments on Animal, Ministry of Culture, Government of India [Registration No. 585/PO/ERe/S/02/CPCSEA]. Colony cages were used to house the animals at an ambient temperature of $20-25^{\circ} \mathrm{C}$ and $45-55 \%$ relative humidity. They had free access to diet and water

Skin samples-The hair from dorsal surface of procured wistar albino rats weighing 120 to $150 \mathrm{~g}$ were carefully trimmed with a pair of scissors and removed by hair removing cream using ether as anesthetic [11]. The clean shaven skin was excised, washed and section of skin was carefully separated from underlying connective tissue with a scalpel and placed in $\mathrm{pH} 6.8$ phosphate buffer solution (PBS) for $1 \mathrm{~h}$ for equilibration before experiment.

\section{Formulation of LBV of imiquimod}

Cold method of preparation for ethosomes [12] was tried to formulate LBV of imiquimod using different excipients and best selected on the basis of maximum percentage entrapment efficiency (\% EE).

\section{Preliminary trials of LBV}

Optimization of phospholipid-Trial ethosomal formulations of imiquimod were investigated for entrapment efficiency (EE) using different phospholipids (phospholipon 90 G (PL 90G), leciva S 70, leciva S 90 and lipoid S 100. PL 90G was selected as the phospholipid for final formulation [13].

Selection and concentration of lactic acid-The amount of lactic acid to be used for formulation was selected by using different concentrations and determining its effect on solubility of imiquimod.

Selection of stabilizer (span 80)-Trial formulations were prepared using stabilizers (span 80 and tween 80 ). Span 80 was selected for final formulation.

\section{Formulations of LBV using central composite design (CCD)}

A Box-Wilson CCD was used for the formulation of LBV. Two independent variables, the amount of lipid: PL 90G (X1) and ethanol (X2) were studied at 3 levels each. The central point $(0,0)$ was studied at quintuplicate. All other processing and formulation variables (imiquimod: $2 \% \mathrm{w} / \mathrm{w}$, lactic acid: $3 \% \mathrm{w} / \mathrm{w}$, span $80: 1.5 \%$ $\mathrm{w} / \mathrm{w})$ were kept constant throughout the study. Table 1 (formulation code F1-F13) gives an account of 13 experimental runs studied, their factor combinations and translation of coded levels to experimental units employed during the study as per the JMP software design expert (12.0.0). The response parameter, \% EE, taken as dependent variable $\mathrm{Y}$ is also shown in same table 1 . The procedure adopted was in following steps:

- PL 90G was dissolved in ethanol and drug added into lactic acid with span 80. Lipid ethanol solution was mixed with drug lactic acid span 80 mixture using vortex mixer.

- Double distilled water was added slowly in a fine stream with constant mixing at $700 \mathrm{rpm}$ with remi mechanical stirrer, in a wellsealed container specially designed.
- Mixing was continued for another 5 min. The mixture was kept at $30{ }^{\circ} \mathrm{C}$ during the preparation and then left to cool at room temperature.

\section{Characterization of LBV formulations}

All the 13 formulations were evaluated for following parameters:

\section{A. Pharmacotechnical characteristics:}

Appearance-Appearance of formulation was observed by visual inspection after cooling.

Determination of $\mathrm{pH}-\mathrm{pH}$ of cooled formulation was determined using Labindia $\mathrm{pH}$ meter. Final $\mathrm{pH}$ was maintained using triethanolamine.

\section{B. Evaluation of response (dependent) parameter (\% EE):}

$\%$ EE in vesicles was measured by dialysis bag method. Dialysis bags containing $1 \mathrm{ml}$ of formulation were immersed in beaker containing $50 \mathrm{ml} 0.1 \mathrm{~N} \mathrm{HCl}$ at room temperature and stirred with a paddle at $37 \pm 0.5{ }^{\circ} \mathrm{C}$ at $100 \mathrm{rpm}$. Dialysis was performed for $5 \mathrm{~h}$. Then, $1 \mathrm{ml}$ aliquote was withdrawn and filtered through a $0.45 \mu \mathrm{m}$ filter membrane, and the concentration of imiquimod in the filtrate was determined by UV spectroscopy [14]. The experiment was performed in triplicate. The $\% \mathrm{EE}$ was calculated using equation (1):

$$
\% E E=\frac{\text { total drug-diffused drug }}{\text { total drug }} \times 100
$$

The results as mean of three independent experiments performed are shown in table 1.

\section{Selection and evaluation of optimized LBV formulation}

Formulation F10 was selected by statistical analysis using JMP software design expert $(12.0 .0)$ for dose titration on the basis of evaluation parameters. $2 \% \mathrm{w} / \mathrm{w}$ of drug-imiquimod was used for formulation of LBV and evaluated for appearance, $\mathrm{pH}$ and $\% \mathrm{EE}$. The average size i.e. size distribution and polydispersity index (PDI) of vesicle formulation was measured using dynamic light scattering (DLS) with a computerized Malvern Autosizer Nano ZS90 inspection system (Malvern Instruments Ltd, Malvern, UK). The system was also used to determine zeta potential of vesicles [15]. Transmission electron microscope (TEM) (JEM-1230, JEOL, Japan) was used as a visualizing aid for the formulation with an accelerating voltage of 80 $\mathrm{kV}$. Samples were negatively stained on a carbon-coated copper grid having $1 \%$ aqueous solution of phosphotungstic acid [16].

Incorporation of optimized LBV formulation containing imiquimod in gel base

Carbopol 980 (NF) forms transparent gel at low concentration with good consistency. It was selected as a gel forming agent due to its non-toxic and non-irritant nature on skin. $2 \%$ carpobol gel base was prepared by soaking it in distilled water overnight. The optimized LBV formulation (equivalent to $2 \%$ drug) was dispersed in gel base with gentle stirring to obtain homogeneity. Gel was neutralized with triethanolamine and mixed properly [17]. For control gel, imiquimod drug $(2 \%)$ was weighed accurately and incorporated into $2 \% \mathrm{w} / \mathrm{w}$ gel base.

\section{Evaluation of vesicle (LBV) and control gel of imiquimod \\ Gel parameters}

Both the gel formulations were tested for appearance, homogeneity (presence of any aggregates) by visual inspection, $\mathrm{pH}$ [11], spreadability percentage drug content [18], viscosity [19], consistency [11] and extrudability [20]. The results were recorded in triplicate for both gels.

\section{In vitro skin permeation study}

These studies were performed on a Franz diffusion cell (FDC) assembly using rat skin. The jacketed cell had two limb reservoirs which comprise of donor and receptor compartment with a sampling port. The diffusion cross sectional area of donor compartment exposed to receptor compartment was $3.14 \mathrm{~cm}^{2}$ with total capacity of receptor compartment $20 \mathrm{ml}$. FDC was assembled 
with skin stuck between donor and receptor compartment. The stratum corneum (SC) surface of skin faced to donor compartment, whereas dermis faced receptor compartment [21]. The receptor compartment was filled with $10 \%$ lactic acid diluted phosphate buffer (pH 6.8) and donor compartment was kept empty. Known quantity of control, vesicle gel ( $2 \% \mathrm{w} / \mathrm{w}$ imiquimod) and marketed formulation ( $5 \% \mathrm{w} / \mathrm{w}$ imiquimod) were spread completely to cover most of the area. The donor chamber and sampling port were covered by aluminum foil to prevent evaporation during study. The receptor fluid was agitated with a magnetic stirrer at a speed of 100 rpm and temperature maintained at $37 \pm 2{ }^{\circ} \mathrm{C}$ by circulating hot water in outer jacket of cell employing a thermostatic water circulator. Aliquots of $1 \mathrm{ml}$ were taken out at predetermined interval and replenished with same volume of receptor fluid to retain the volume in receptor compartment at constant level. Samples were suitably diluted and analyzed for imiquimod by UV-visible spectroscopy at $\lambda_{\max } 227 \mathrm{~nm}$. The experiment was performed six times for each formulation.

\section{Drug retention study}

At the completion of experiment i.e. $24 \mathrm{~h}$, drug retained in layers of skin was measured for control, vesicle gel and marketed formulation. The skin surface was cleaned five times to remove formulation with cotton ball drenched with fresh PBS ( $\mathrm{pH}$ 6.8). A scissor was used to cut the skin into small pieces. The skin in cut sections was homogenized with methanol and then sonicated for 20 min to extract drug. The tissue suspensions were filtered and analysed by HPLC using C8 column and UV detection at $227 \mathrm{~nm} \mathrm{[22].}$ The method was validated for linearity, accuracy, precision, LOD and LOQ. The linearity of measurement was from $0.05-0.5 \mu \mathrm{g} / \mathrm{ml}\left(\mathrm{R}^{2}\right.$ 0.9952) [23]

\section{In vivo skin permeation study}

In vivo skin permeation study was carried out using procured wistar albino rats 6 to $8 \mathrm{w}$ old weighing 120 to $150 \mathrm{~g}$. The rats were divided into three groups, six in each. Rat dorsal skin was shaved carefully to remove hair. Then, known quantity of control, vesicle gel and marketed formulation was topically applied onto the test area. At the end of experiment $(24 \mathrm{~h})$, the rats were gently sacrificed. The complete thick skin tissue was excised and rinsed in PBS (pH 6.8) to clear off residual preparation. The removed skin after excision was then processed as described for in vitro studies, and subsequently analyzed by HPLC [24].

\section{Local accumulation efficiency (LAE) and permeation flux}

LAE values were calculated as the ratio of drug accumulated into skin/drug delivered through skin in vitro [24]. Permeation flux (permeation rate) at steady state $\left(\mathrm{J}, \mu \mathrm{gcm}^{-2} \mathrm{~h}^{-1}\right)$ and lag time $\left(\mathrm{T}_{\text {lag }}, \mathrm{h}\right)$ were calculated from slope and x-intercept respectively of linear portion of curve by plotting cumulative amount of imiquimod permeated through skin vs. time [25].

\section{Evaluation of bilayer fluidity using fourier transform infra-red (FTIR) spectroscopy}

On the basis of previous methods reported, whole skin, epidermis and SC were prepared. wistar albino rats (either sex, weighing 120 to $150 \mathrm{~g}$ ) were taken, sacrificed and dorsal skin excised after hair removal. The excised skin was washed with water and subcutaneous tissue removed surgically. The skin was defrosted $\left(-20^{\circ} \mathrm{C}\right)$, till use after washing with PBS. The epidermis was prepared by treating skin with $1 \mathrm{M}$ sodium bromide solution in water for $12 \mathrm{~h}$. SC with side facing upward was separated from epidermis using $0.1 \% \mathrm{w} / \mathrm{v}$ trypsin solution in water for $12 \mathrm{~h}$. Finally, desiccators were used to completely dry SC sheets. On the day of experiment, sheets were treated with different formulations (control, vesicle gel, and marketed formulation) for $8 \mathrm{~h}$, dried and investigated by FTIR spectrophotometer between 4000 and $1000 \mathrm{~cm}^{-1}$ [26].

\section{Statistical analysis}

Data calculated was mean \pm standard deviation (SD) from at least six independently conducted experiments. The statistical analysis was done on Graph Pad Prism version 7.0 using One-way ANOVA (multiple comparison) and a value of $\mathrm{P}<0.05$ was considered statistically significant.

\section{Skin irritancy test}

This is a skin safety test to determine possible localized reaction of optimized vesicle formulation on skin. Erythema and edema are manifestations of dermal irritation. Extent of development of erythema and edema were indicated on the basis of grading of dermal response using Draize grading scale [27]: no erythema and edema-0, very slight erythema and edema-1, well defined erythema but slight edema-2, moderate to severe erythema with moderate edema-3 and severe erythema with severe edema-4.

Wistar albino rats, weighing 150-200 g were acclimatized for $7 \mathrm{~d}$ to laboratory conditions prior to experiments and kept on a synthetic pellet diet and water ad libitum. The hair from dorsal surface of rats was removed with a hair removing cream, taking care not to damage SC. Four hours prior to the experiment, animals were divided into three groups, containing 6 rats in each group: Group 1: control gel; Group 2: vesicle gel; and Group 3: marketed formulation. The formulations were applied 3 times a week for one week (7 d) to roughly around $3 \mathrm{~cm}^{2}$ area of skin and animals returned to their cages. The application area was examined for dermal reaction (erythema) at $24 \mathrm{~h}$ and $48 \mathrm{~h}$ after the formulation has been applied [28].

\section{Skin histological examination}

Alteration in histology and compliance of skin was evaluated using wistar albino rats after application of different formulations (control, vesicle gel and marketed preparation). The hair from dorsal surface of rats was removed with a hair removing cream, taking care not to damage SC. Then known quantity of each formulation was applied to the skin free from hair with uniform spreading within selected area. Autopsy samples of skin were taken from rats of all groups and $10 \% \mathrm{v} / \mathrm{v}$ formalin solution used for fixation for $24 \mathrm{~h}$. Paraffin bees wax tissue blocks were prepared for sectioning at $10 \mu \mathrm{m}$ by slide microtome. The collected tissue sections were put on glass slides and hematoxylin and eosin stains used for staining. The stained sections were examined histopathologically through a light microscope [24].

\section{Stability studies}

The ability of vesicles to retain drug imiquimod was assessed by keeping vesicle gel formulation at different temperatures, i.e., $4 \pm 2{ }^{\circ} \mathrm{C}$, $25 \pm 2{ }^{\circ} \mathrm{C}$ (room temperature, RT), and $45 \pm 2{ }^{\circ} \mathrm{C}$ for different periods of time $(30,60$ and $90 \mathrm{~d})$. The gel formulations were kept in glass vials (10 ml capacity) covered with aluminum foils. The stability was assessed quantitatively by monitoring $\mathrm{pH}$, homogeneity, spreadability and percentage drug content of vesicles over time. Stability studies for the formulation were carried out in triplicate [29].

\section{RESULTS AND DISCUSSION}

Dermal delivery of any drug poses significant challenge because of obstinate barrier properties of SC. Many approaches are gaining interest to cross this skin obstruction including use of LBV to amend the SC. Inclusion of ethanol in ethosomes is an excellent approach to fluidize the skin lipid membrane as it intermingles with the polar head of lipid molecules and reduces the melting point of SC lipid, thereby increasing lipid fluidity and cell membrane permeability [30]. But, the sole use of ethanol may lead to too much transdermal flux, and consequently, insufficient skin retention [31]. The formulation of imiquimod presents a peculiar challenge due to its insoluble nature in most of the solvents except fatty acids [8]. Therefore, lactic acid has been used to solubilize imiquimod. Literature suggests that ethanol in combination with surfactant in vesicles can considerably boost the skin retention of drugs [32]. The surfactant, span 80 could penetrate into skin lipids due to its long lipophilic hydrocarbon chain, untie the intermolecular binding among SC lipids, and increase the skin permeability. Thus, LBV formulated using lactic acid and span 80 in carbopol gel base could certainly improve the dermal delivery of encapsulated imiquimod.

\section{Formulation of LBV of imiquimod}

\section{Preliminary trials of LBV}

Selection of lipid-PL 90G was selected on the basis of maximum \% EE obtained. 
Selection and concentration of lactic acid-The formulation of imiquimod presents a particular challenge due to its insolubility in aqueous solutions at physiological pH. It is soluble in acidic aqueous solutions and its $0.1 \mathrm{M}$ lactic acid-based formulations are in clinical development for the treatment of bladder cancer. Lactic acid is a widely used pharmaceutical excipient. Therefore, it was hypothesized that vesicles having lactic acid in core would make possible encapsulation of soluble imiquimod while enabling the bulk aqueous phase external to vesicles to maintain a physiological buffer at close to a neutral $\mathrm{pH}$, which is desirable to minimize skin irritation [8]. 3\% $\mathrm{w} / \mathrm{w}$ concentration of lactic acid was chosen for final formulation.

Selection of stabilizer (span 80)-Imiquimod, a basic drug with pka value 2.7 easily dissolves in acidic medium but on increasing the $\mathrm{pH}$ of media, drug precipitates out. Drug has highest solubility in lactic acid but low solubility in ethanol as well as phospholipid-ethanol solution. When lactic acid is used as solubilizing agent for formulation of vesicles of imiquimod, it reduced the $\mathrm{pH}$ of formulation around 3 . This $\mathrm{pH}$ changes integrity of lipid bilayer to some extent. In addition, the $\mathrm{p} / \mathrm{H}$ of final formulation has to be adjusted around 7 , a requirement for topical drug delivery system to be non-irritating to skin. Therefore, to maintain $\mathrm{pH}$ of final formulation as well as to reduce precipitation of drug, surfactant span 80 [24] was used for the formulation of vesicles. On addition of span 80, the formulation had approx. $\mathrm{pH} 4$ and precipitation of drug reduced on increasing the $\mathrm{pH}$ to 6-7. This surfactant, span 80 having low HLB value 4.3 (lipophillic) favors vesicle formation and entrapment of drug. When hydrophilic surfactants (tween 80) are considered, their hydrophilicity on hydration do not permit them to attain a concentrated system and inhibit the free hydrated units to exist as aggregates and coalesced to form lamellar structure. Surface free energy also decreases with high HLB value surfactant resulting in formation of vesicles of bigger size. High HLB value surfactants (hydrophilic) do not allow vesicle formation due to their high aqueous solubility as in the case of tween 80 (HLB 15.0) [33]. 1.5\% $\mathrm{w} / \mathrm{w}$ concentration of span 80 was chosen for final formulation.

\section{Formulation and characterization of LBV of imiquimod using CCD}

Cold method used for preparation of ethosomes was quite effective in the formulation of LBV of imiquimod but lactic acid and span 80 have been used in addition to PL 90G and ethanol. All formulations were found to be translucent and final $\mathrm{pH}$ is given in table 1 .

Table 1: Composition of different LBV formulations of imiquimod as per CCD with results

\begin{tabular}{|c|c|c|c|c|c|}
\hline \multirow{2}{*}{$\begin{array}{l}\text { S. } \\
\text { No. }\end{array}$} & \multirow[t]{2}{*}{ Formulation code } & \multicolumn{2}{|c|}{ Independent variable } & \multirow[t]{2}{*}{ Final pH } & \multirow{2}{*}{$\begin{array}{l}\text { Dependent variable } \\
\text { Y Percentage entrapment efficiency* }\end{array}$} \\
\hline & & $\begin{array}{l}X_{1} \text { PL 90G } \\
(\% \mathrm{w} / \mathrm{w}) \\
\end{array}$ & $\begin{array}{l}\mathrm{X}_{2} \text { Ethanol } \\
(\% \mathrm{w} / \mathrm{w})\end{array}$ & & \\
\hline 1 & F1 & 3 & 30 & 6.8 & $80.33 \pm 0.10$ \\
\hline 2 & $\mathrm{~F} 2$ & 3 & 44.1421 & 6.9 & $44.60 \pm 0.23$ \\
\hline 3 & F3 & 2 & 40 & 7.0 & $64.86 \pm 0.28$ \\
\hline 4 & $\mathrm{~F} 4$ & 3 & 30 & 6.8 & $79.89 \pm 0.17$ \\
\hline 5 & F5 & 1.58579 & 30 & 6.6 & $67.01 \pm 0.06$ \\
\hline 6 & F6 & 3 & 15.8579 & 6.4 & $79.33 \pm 0.25$ \\
\hline 7 & F7 & 3 & 30 & 6.8 & $79.56 \pm 0.00$ \\
\hline 8 & F8 & 4.41 & 30 & 6.8 & $55.63 \pm 0.37$ \\
\hline 9 & F9 & 4 & 40 & 7.0 & $57.02 \pm 0.77$ \\
\hline 10 & F10 & 3 & 30 & 6.9 & $79.64 \pm 0.29$ \\
\hline 11 & F11 & 4 & 20 & 6.8 & $78.06 \pm 0.17$ \\
\hline 12 & F12 & 3 & 30 & 7.0 & $79.42 \pm 0.35$ \\
\hline 13 & F13 & 2 & 20 & 6.6 & $75.39 \pm 0.42$ \\
\hline
\end{tabular}

*Values $[$ mean $\pm \operatorname{SD}(n=3)]$

$\%$ EE was determined using dialysis bag method and data given in table 1. Encapsulation of drug within a vesicular system, as measured by EE, is a key parameter that evaluates the potentiality of a delivery system. The value of EE determined for vesicles is satisfactory, indicating that PL 90G, lactic acid, ethanol and span 80, used for formulation enhanced entrapment of drug. Further, presence of lactic acid would allow for better solubility of imiquimod, improving its distribution throughout the vesicle, as compared to it being limited to core as in traditional liposomes [34] Span 80, with increased carbon chain length of surfactant increases lipophilicity and solubility of lipophilic drug in bilayer which might explain high \% EE of imiquimod in bilayer of vesicles [24] Moreover, vesicles containing higher amounts of ethanol have been found with lean membranes, related to creation of a phase through interpenetrating hydrocarbon chains, which finally improved EE of lactic acid solubilized imiquimod in vesicles [35].

\section{Optimization of formulation using CCD}

Evaluation of results by regression analysis: The observed value of response (\% EE) was further analyzed statistically to evaluate effect of various factors and interaction of factors using DOE. Data were analyzed by JMP software 12.0 .0 as:

- Actual vs. predicted plot

- Prediction profiler

- Counter plot

The actual vs. predicted plot (fig. $1 \mathrm{~A}$ ) shows $\mathrm{R}^{2}$ and $\mathrm{P}$ value 0.90 and 0.0023 respectively. This means that prediction model for response EE is significant.

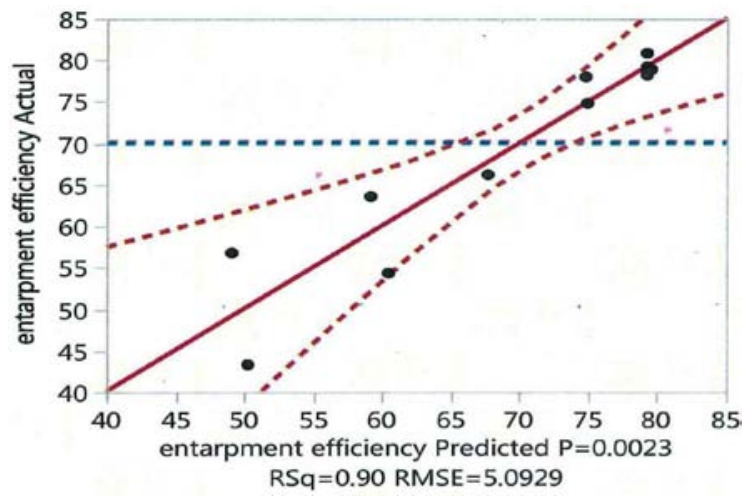

Fig. 1A: Actual vs. predicted plot for response EE

The prediction profiler changes one variable at a time keeping other factors constant. Prediction profiler as shown in fig. 1B predicts that there is an optimum concentration (30\%) of ethanol and a range of concentration (2.5 to 3.5\%) of PL90 G for maximum EE. The selected concentrations of PL90 G and ethanol were found to be best for LBV formulation to show maximum results.

The contour plot was plotted between amount of PL 90 G and ethanol as shown in fig. 1C.

- On the basis of desirability function best formulation was predicted by model using ethanol (30\%) and PL 90 G (3\%). 
- Predicted \% EE (response) of optimized formulation was $79.228 \%$.
F10 formulation optimized as predicted by counter profiler was fabricated.

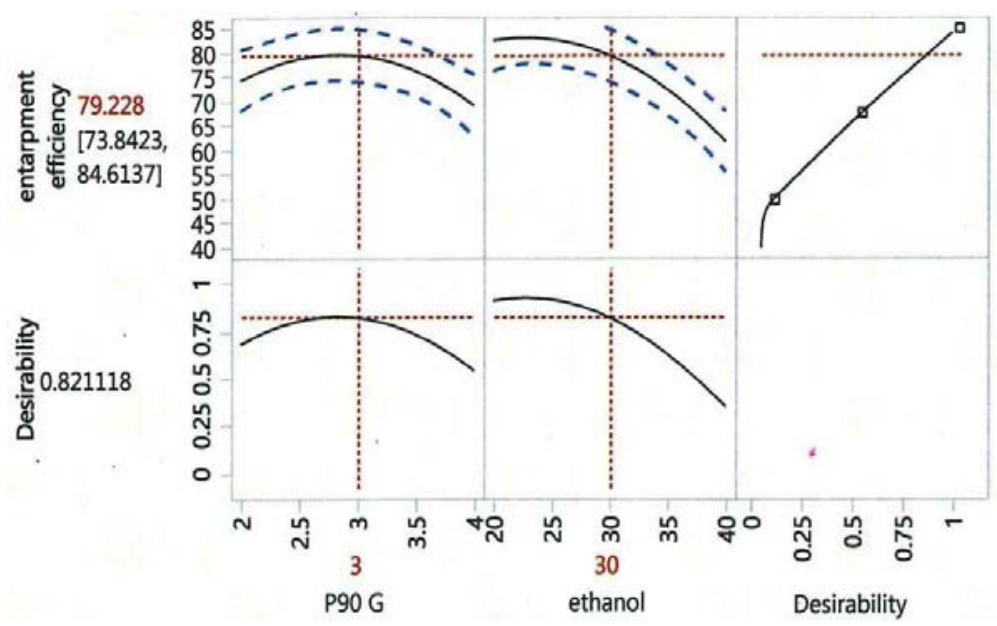

Fig. 1B: Prediction profiler

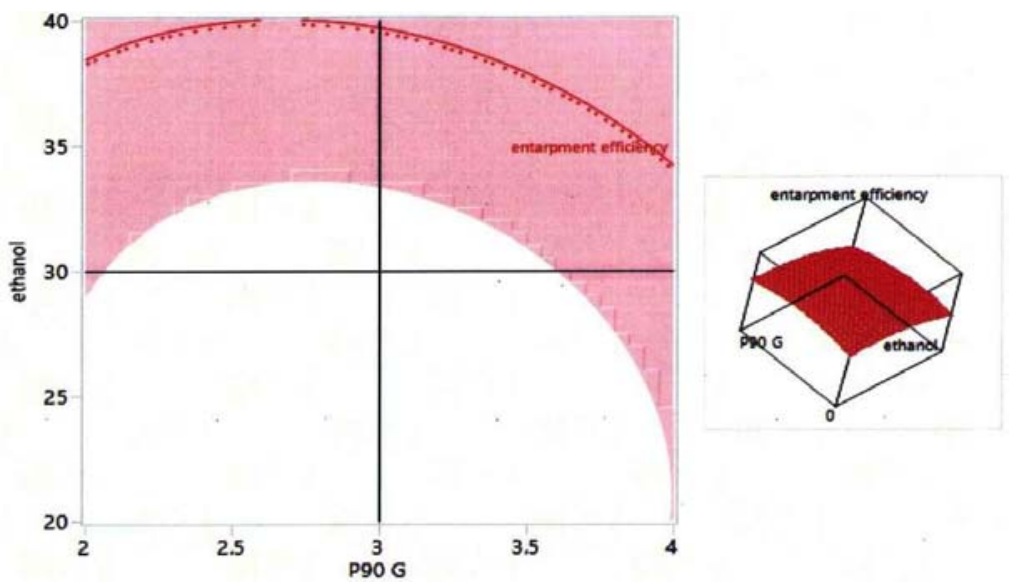

Fig. 1C: Contour plot and 3D response surface plot showing the relationship between various levels of two factors on EE

\section{Mathematical modeling}

Mathematical relationship generated using multiple linear regression analysis for the studied response variable is expressed as equation (2):

$\% \mathrm{EE}=+79.228-2.550 \mathrm{X}_{1}-10.359 \mathrm{X}_{2}-2.485 \mathrm{X}_{1} \mathrm{X}_{2}-7.58 \mathrm{X}_{1}{ }^{2}-7.16 \mathrm{X}_{2}{ }^{2}$.

The polynomial equation comprised coefficient for intercept, firstorder main effect, interaction term and higher order effect. The sign and magnitude of main effect signified the relative influence of each factor on response i.e. \% EE. The polynomial equation (2) for \% EE denoted that both coefficients $\mathrm{X}_{1}$ and $\mathrm{X}_{2}$ bear a negative sign. Therefore, increasing the concentration of both lipid and ethanol were expected to decrease \% EE. This was further revealed by response surface plots as shown in fig. 1C.

\section{Selection and evaluation of optimized vesicle (LBV) formulation}

F10 formulation containing $2 \% \mathrm{w} / \mathrm{w}$ of drug imiquimod selected for dose titration was found to be translucent with $\mathrm{pH} 7.01 \pm 0.02$ and \% EE 88.27 \pm 0.45 . The net charge on vesicles is an important parameter that can influence vesicular properties such as stability as well as skin-vesicle interaction. The optimized vesicle formulation was found to have particle size $394.8 \pm 9.6 \mathrm{~nm}$ (fig. 2A) and zeta potential$16.5 \pm 2.5 \mathrm{mV}$ (fig. $2 \mathrm{~B}$ ), desired results indicating good stability of formulation. Apart from ethanol (makes negative charge on vesicles), span 80 also exerts effect on globule size. Desired particle size of vesicles might be due to its surface active properties (provides elasticity) and membrane softening/reduction ability [13]. TEM study (fig. 3) clearly confirms the formation of vesicular structure with spherical shape. The optimized formulation has shown imperfect round shape explaining fluidized effect of ethanol as observed earlier [26].

\section{Evaluation of vesicle (LBV) and control gel of imiquimod \\ Gel parameters}

The gel formulation was found to be transparent, homogenous with $\mathrm{pH}$ in the range of 6.9-7.2 within the normal skin $\mathrm{pH}$ in healthy people which is considered to be acceptable for avoiding risk of irritation upon application to skin [19]. The data of spreadability indicates that the gel is easily spreadable by a small amount of shear [26]. Viscosity of control gel is more as compared to vesicle gel and viscosity of vesicle gel within permissible limit. Vesicle gel did not break at different rpm which represents its mechanical strength. Carbopol 980 forms a physically bonded network by formation of junction zones responsible for mechanical strength of gel [19]. Consistency reflects the ability of gel to get expelled in consistent and preferred amount when tube is squeezed [11] and extrudability measures force required to expel gel from tube [20]. The values obtained (table 2) are positive for vesicle gel. 


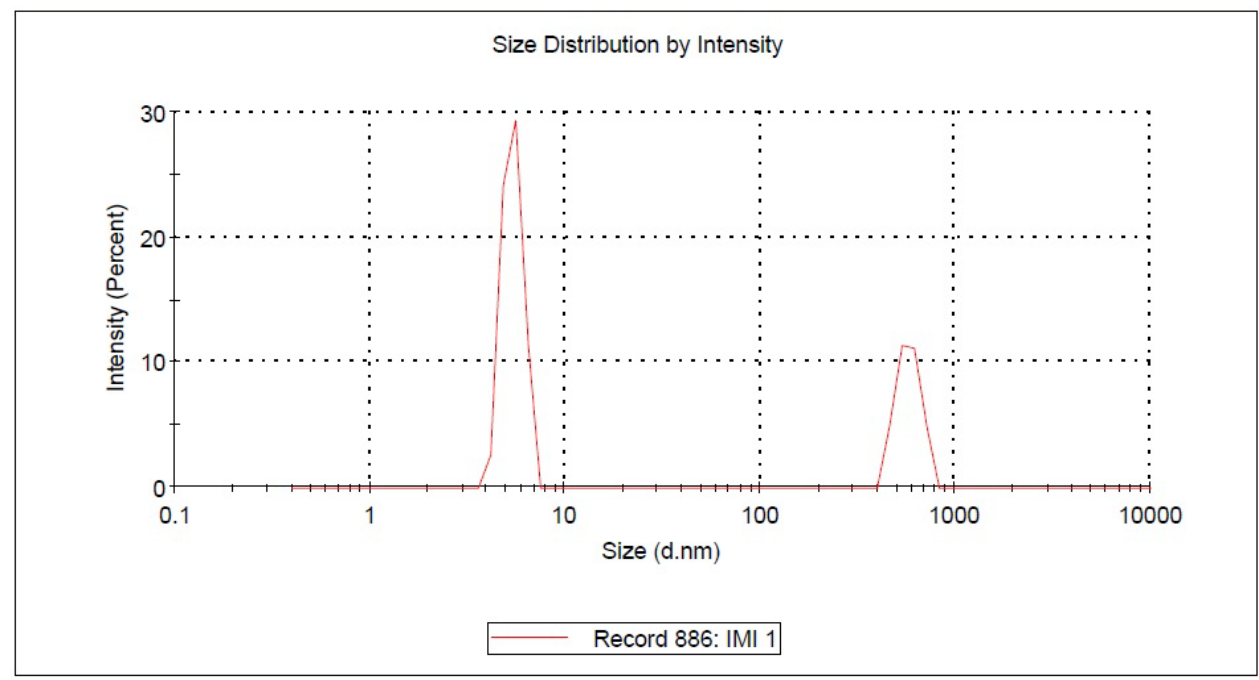

Fig. 2A: Particle size of optimized LBV

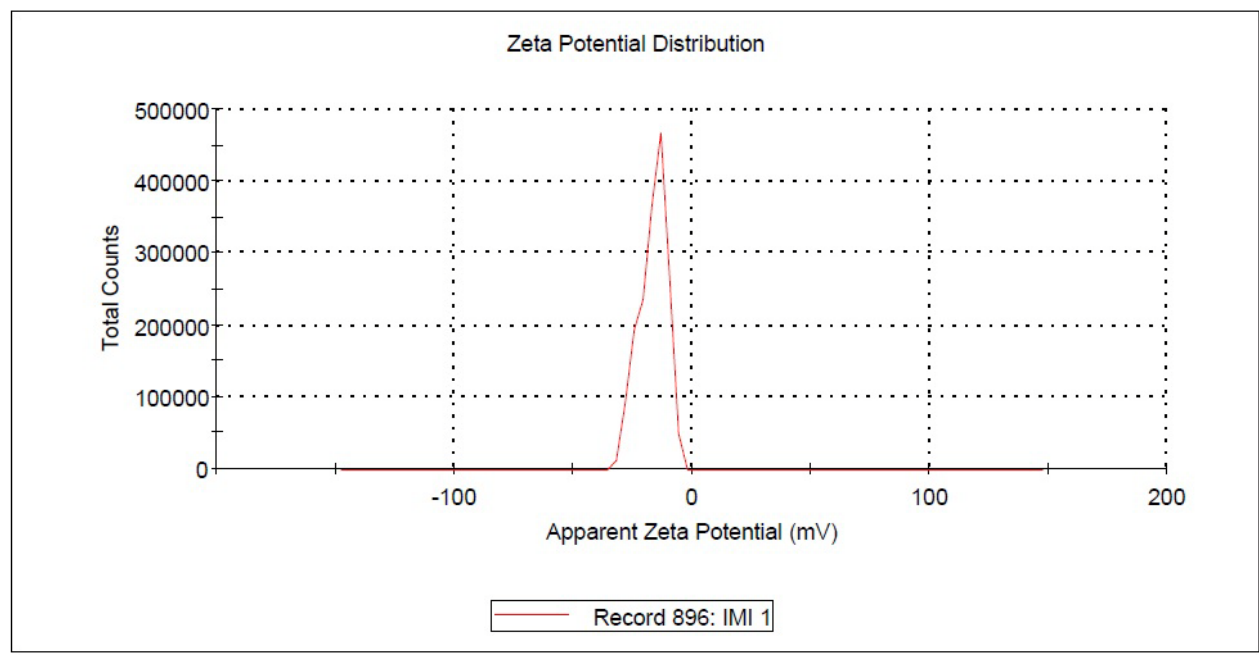

Fig. 2B: Zeta potential of optimized LBV

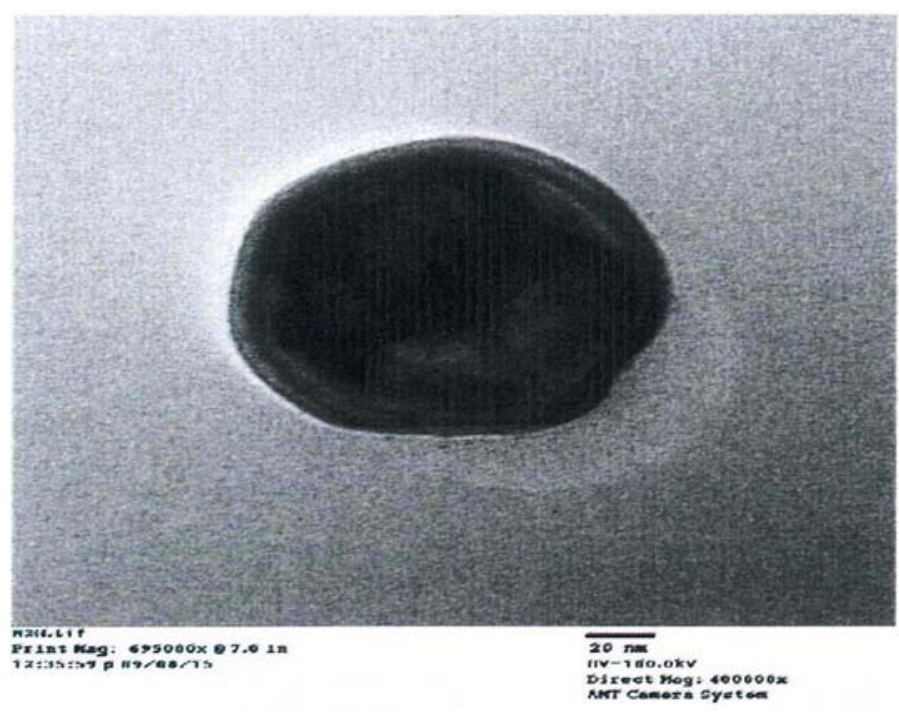

Fig. 3: TEM of optimized LBV 
Table 2: Spreadability, percentage drug content, viscosity, consistency and extrudability for vesicle and control gel formulation

\begin{tabular}{llllll}
\hline Formulation & $\begin{array}{l}\text { Spreadability } \\
\text { (gcm/sec) }\end{array}$ & $\begin{array}{l}\text { Percentage drug content } \\
\text { (\%) }\end{array}$ & Viscosity (cp) & Consistency (mm) & Extrudability (g/cm ${ }^{\mathbf{2}}$ ) \\
\hline Vesicle gel & $14.3 \pm 0.34$ & $94.3564 \pm 0.43$ & $13500 \pm 1.67$ & $6.1 \pm 0.14$ \\
Control gel & $10.6 \pm 0.44$ & $90.414 \pm 0.36$ & $26230 \pm 1.34$ & $5.7 \pm 0.15$ & $16.47 \pm 0.11$ \\
\hline
\end{tabular}

Values [mean $\pm \operatorname{SD}(n=3)]$

\section{In vitro skin permeation study}

Percentage cumulative drug release (\% CDR) of control gel, vesicle gel and marketed formulation represented in fig. $4 \mathrm{~A}$ illustrate that both vesicle gel and marketed formulations displayed sustained permeation of imiquimod during the first $12 \mathrm{~h}$. With the addition of lactic acid and span 80 , permeation amount of drug was remarkably lower than that of control gel. Moreover, the maximum \% CDR at 24 h was only $10.13 \%$ from vesicle gel which demonstrated that more active agent retained in epidermis and dermis.

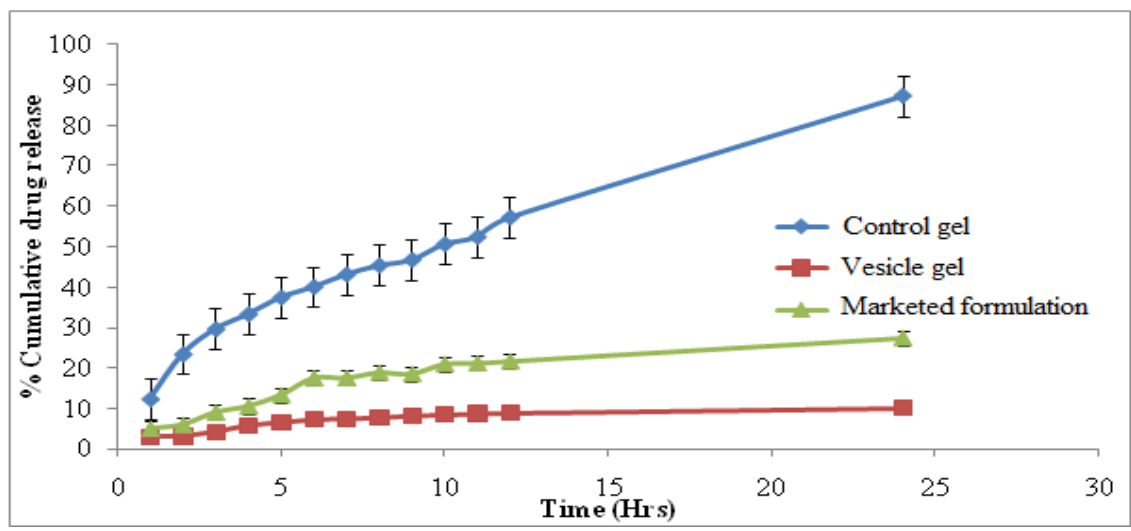

Fig. 4A: In vitro percentage cumulative drug release plot with control gel, optimized vesicle gel and marketed formulation. Data represents mean $\pm S D$ of six experiments

\section{Drug retention study}

Fig. 4B represents the cumulative amount of drug retained into skin layers after performing in vitro permeation study for $24 \mathrm{~h}$. The drug deposition significantly increased in skin layer by vesicle gel as compared to control gel and marketed formulation. Extra drug deposited into epidermal and dermal layers due to the presence of reservoir of lactic acid solubilized imiquimod in vesicles. The improvement in dermal delivery of drug due to LBV had been related to a number of different mechanisms such as alteration of skin hydration and increased thermodynamic activity of drug. This result also indicated that vesicles had targeted effect to enhance drug deposition in SC and deeper skin stratum, meanwhile reduce drug permeation into blood, thus avoiding systemic side effects [24]. From the results, it can be concluded that LBV as a carrier for imiquimod not only enhanced penetration but also promoted more retention of drug within the skin [36]. In short, in vitro permeation studies revealed superiority of vesicle gel as compared to control gel and marketed formulation.

\section{In vivo skin permeation study}

This study further conducted to find the retention of drug in skin layers showed same trend as in vitro skin permeation results. The retention of drug in skin layer (fig. 4B) was significantly higher for vesicle gel might be due to depot formation of lactic acid solubilized imiquimod. Moreover, the improved skin permeability of vesicles (like ethosomes) may be related to its deformable nature. Therefore, the modified LBV of imiquimod had potential to deliver effective amount of drug to targeted site in skin, maintenance of its depot and prolong residence time for treatment of genital warts

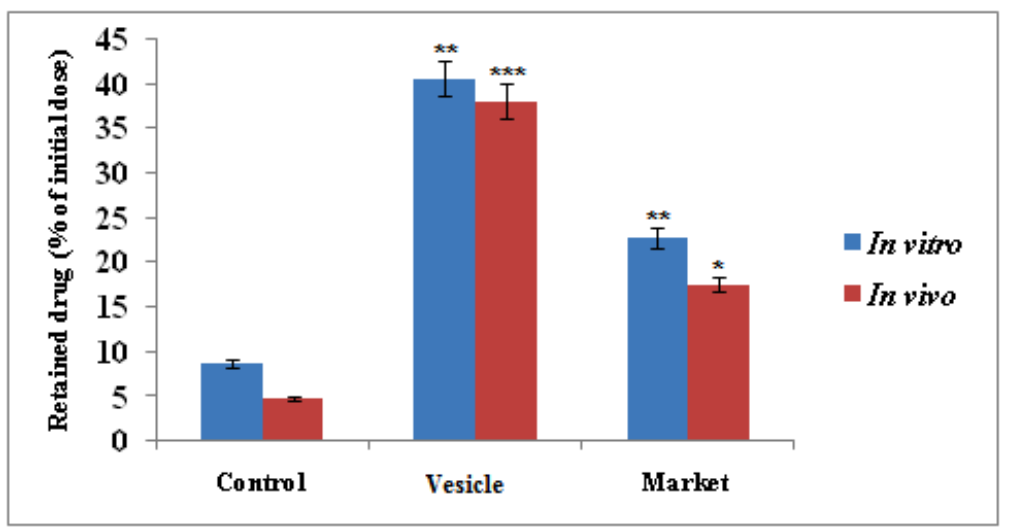

Fig. 4B: Imiquimod retained in skin layers of in vitro and in vivo skin permeation study after $24 \mathrm{~h}$ with control gel, optimized vesicle gel and marketed formulation. Data represents mean $\pm \mathrm{SD}$ of six experiments $\left({ }^{*} \mathrm{P}<0.5,{ }^{* * \mathrm{P}}<0.01,{ }^{* * *} \mathrm{P}<0.001\right)$ 


\section{LAE and permeation flux}

LAE [24] values as given in table 3 show highest value (4.021) for vesicle gel followed by marketed formulation (0.8314) and control gel $(0.1008)$ suggesting skin targeted effect of imiquimod-loaded vesicle gel. The relatively higher drug retention into skin layer indicates that LBV were capable of penetrating into hairless rat skin, reaching the target site of dermis where they formed a depot from which drug could be released. Permeation flux (permeation rate) [25] at steady state $\left(\mathrm{J}, \mu \mathrm{gcm}^{-2} \mathrm{~h}^{-1}\right)$ values given in table 3 indicate low value for vesicle gel followed by marketed formulation and control gel. This suggests that vesicles of imiquimod did not permeate into systemic circulation. Lag time $\left(\mathrm{T}_{\text {lag }}, \mathrm{h}\right)$ obtained for all the formulations was almost same indicating no difference in onset of action.

Table 3: Results of in vitro permeation study from control gel, vesicle gel, and marketed formulation

\begin{tabular}{|c|c|c|c|c|}
\hline \multirow{2}{*}{$\begin{array}{l}\text { Formu- } \\
\text { lation }\end{array}$} & \multicolumn{2}{|c|}{ Percentage of initial dose } & \multirow[t]{2}{*}{ Local accumulation efficiency } & \multirow{2}{*}{$\begin{array}{l}\text { Permeation flux } \\
\left(\mathrm{J}, \mu \mathrm{gcm}^{-2} \mathrm{~h}^{-1}\right)\end{array}$} \\
\hline & Drug permeated & Drug retained & & \\
\hline Control gel & $87.17 \pm 1.662$ & $8.755 \pm 0.1831$ & $0.1008 \pm 0.002513$ & $0.3075 \pm 0.07431$ \\
\hline Vesicle gel & $10.13 \pm 0.5795^{* *}$ & $40.67 \pm 0.09768^{* *}$ & $4.021 \pm 0.2292^{* *}$ & $0.07932 \pm 0.01806^{* *}$ \\
\hline Marketed & $27.46 \pm 1.237^{* * *}$ & $22.79 \pm 0.2808^{* *}$ & $0.8314 \pm 0.0300 *$ & $0.1764 \pm 0.02759 *$ \\
\hline
\end{tabular}

Values as mean $\pm \mathrm{SD}$ of six experiments $\left({ }^{*} \mathrm{P}<0.5,{ }^{* *} \mathrm{P}<0.01,{ }^{* * *} \mathrm{P}<0.001\right)$

\section{Evaluation of bilayer fluidity using FTIR spectroscopy}

FTIR analysis provided more spectral information on lipid vesicles-skin interaction. In IR spectrum, intensity of band (height and area) indicated the quantity of lipids/proteins in SC. When an enhancer extracted lipids of SC, these bands would show a decrease in band height and/or area or would completely disappear [24] Alteration in SC fluidity was noted by concentrating in area near to $2850 \mathrm{~cm}^{-1}$ and $2920 \mathrm{~cm}^{-1}$. After treatment of skin with optimized vesicle gel, peaks obtained near at $2850 \mathrm{~cm}^{-1}$ and $2920 \mathrm{~cm}^{-1}$ due to $\mathrm{C}-\mathrm{H}$ symmetric stretching and $\mathrm{C}-\mathrm{H}$ asymmetric stretching absorbance (untreated skin) were observed with slight alterations as compared to control and marketed formulation shown in table 4 [26].

Table 4: Alterations on the $\mathrm{C}-\mathrm{H}$ symmetric and $\mathrm{C}-\mathrm{H}$ asymmetric stretching absorbance shifts on acyl chains of stratum corneum lipids upon application with control gel, vesicle gel and marketed formulation

\begin{tabular}{lll}
\hline Skin treatments & C-H symmetric stretching $\left.\mathbf{( c m}^{-1}\right)$ & C-H asymmetric stretching $(\mathbf{c m}-\mathbf{1})$ \\
\hline Untreated & $2850.84 \pm 0.5784$ & $2920.78 \pm 0.6204$ \\
Control gel & $2850.01 \pm 0.8886^{*}$ & $2918.71 \pm 2.2510^{*}$ \\
Vesicle gel & $2854.42 \pm 0.5958^{* * *}$ & $2924.38 \pm 0.5172^{* * *}$ \\
Marketed & $2852.13 \pm 1.089^{* *}$ & $2920.09 \pm 0.4294^{* *}$ \\
\hline
\end{tabular}

Each value represents mean $\pm \mathrm{SD}$ of six experiments $\left({ }^{*} \mathrm{P}<0.5,{ }^{* *} \mathrm{P}<0.01,{ }^{* * *} \mathrm{P}<0.001\right)$

\section{Skin irritancy test}

The possibility of skin irritation of any formulation could limit its use and patient acceptability. Thus, it was reasonably essential to conduct cumulative skin irritation test of vesicle gel formulation on animal skin for $7 \mathrm{~d}$ and compare with control gel and marketed formulation. The results of dermal irritation experiments are summarized in table 5 .

Table 5: Dermal response from control gel, vesicle gel and marketed formulation in terms of mean erythemal score

\begin{tabular}{llll}
\hline Day of application & Mean erythemal score & & Marketed formulation \\
\cline { 2 - 4 } & Control gel & Vesicle gel & 0.00 \\
\hline Day 1 & & & 0.50 \\
$24 \mathrm{~h}$ after application & 0.00 & 0.00 & 1.00 \\
$48 \mathrm{~h}$ after application & 0.50 & 0.33 & 1.11 \\
Day 3 & 1.33 & 0.50 & 1.33 \\
$24 \mathrm{~h}$ after application & 1.50 & 0.67 & 1.50 \\
$48 \mathrm{~h}$ after application & & & \\
Day 5 & 1.83 & 0.83 & 1.00 \\
24 h after application & 2.17 & & \\
\hline 8 h after application & &
\end{tabular}

No significant dermal response, including erythema and edema, were found in rats treated with vesicle gel as compared to control gel and marketed formulation of imiquimod. Mean erythemal scores of drug-loaded vesicle gel were quite low which may be due to addition of carbopol which increased viscosity and its three dimensional network structure reduced chances of direct contact of imiquimod with skin layers. Thus, LBV of lactic acid solubilized imiquimod may possess remarkable benefits over control gel and marketed formulation in terms of patient acceptance and tolerability for the treatment of genital warts [28]

\section{Skin histological examination}

The histopathological examination of treated dorsal skin of wistar albino rats with control gel, vesicle gel and marketed formulation was investigated to evaluate the biocompatibility of formulation. Histopathological images as shown in fig. 5 had no significant thickening of skin layers and rat epidermis remained intact with no obvious changes. No inflammatory reaction was seen in skin treated with vesicle gel as observed earlier (24) indicating that the imiquimod loaded LBV is biocompatible. Further, histological images of rat skin tissue treated with all the three formulations could not be differentiated on observation under light microscope. The results confirmed that using phospholipids in conjunction with ethanol, lactic acid and span 80 hardly had any irritation of skin with short time application.

\section{Stability studies}

The results of accelerated stability studies are summarized in table 6. Vesicle gel stored for $90 \mathrm{~d}$ at different temperature showed no 
change in appearance suggesting its stability under given conditions. No significant variation in $\mathrm{pH}$ and spreadability was observed after subjecting the formulation to stability stress for 3 mo. The decrease in $\mathrm{pH}$ observed may be due to presence of lactic acid in the formulation as it has been reported that $\mathrm{pH}$ value gradually reduced by-0.4 units after one month of manufacture [9]. The Imiquimod loaded vesicle gel formulation was also evaluated for percentage drug content at different temperatures. The outcome suggested greater drug loss at elevated temperatures after 3-month period suggesting cold storage for formulation.

\section{Control}

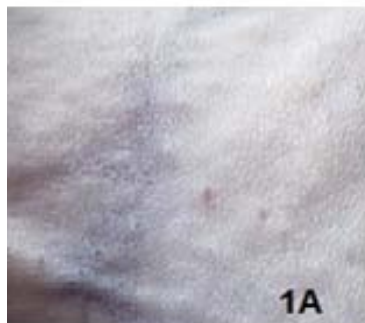

$1 \mathrm{~A}$

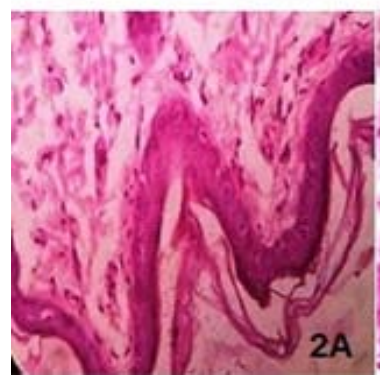

Vesicle

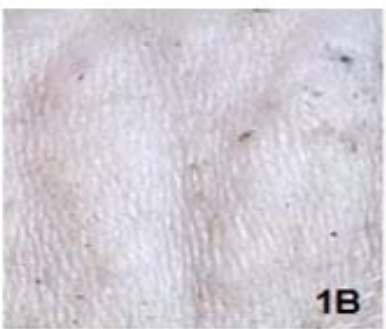

$1 B$
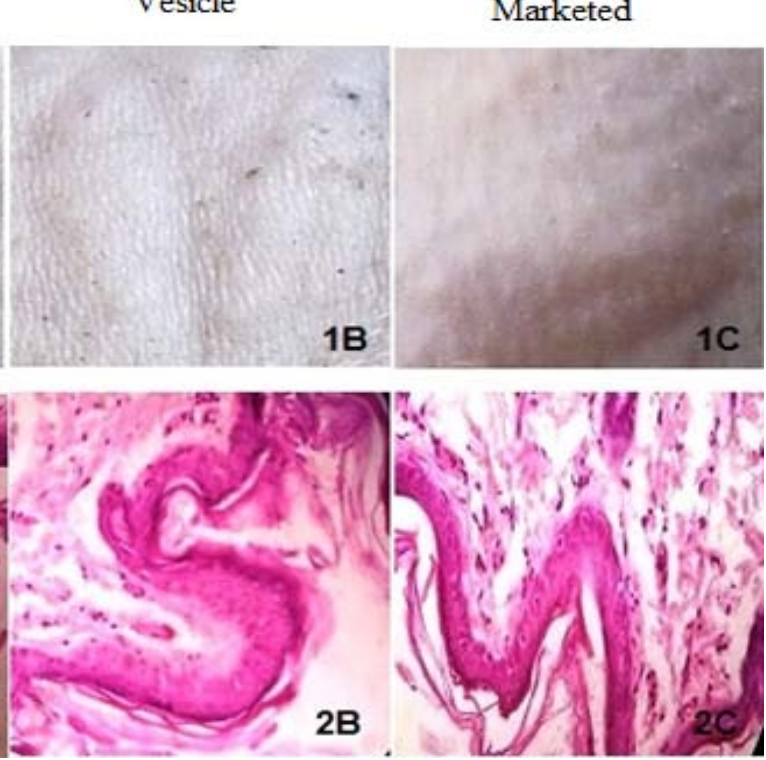

Fig. 5: Wistar rat (1) dorsal skin appearance and (2) histological images of skin tissue after treatment with (A) control gel (B) vesicle gel and (C) marketed formulation

Table 6: Stability study data of vesicle gel formulation

\begin{tabular}{llllll}
\hline $\begin{array}{l}\text { Storage condition } \\
\text { (Temp.) }\end{array}$ & Storage time (Days) & $\mathbf{p H}$ & Homogeneity & $\begin{array}{l}\text { Spreadability } \\
\text { (gcm/sec) }\end{array}$ & Percentage drug content at 244 nm \\
\hline Initial & 0 & $6.8 \pm 0.045$ & Homogenous & $14.30 \pm 0.34$ & $108.68 \pm 0.72$ \\
$4 \pm 2{ }^{\circ} \mathrm{C}$ & 30 & $6.5 \pm 0.076$ & Homogenous & $14.20 \pm 0.78$ & $107.58 \pm 0.005$ \\
& 60 & $6.3 \pm 0.14$ & Homogenous & $13.95 \pm 0.004$ & $99.09 \pm 0.054$ \\
& 90 & $6.0 \pm 0.76$ & Homogenous & $12.35 \pm 0.23$ & $97.51 \pm 0.0045$ \\
$25 \pm 2{ }^{\circ} \mathrm{C}$ & 30 & $6.0 \pm 0.097$ & Homogenous & $14.10 \pm 0.34$ & $103.44 \pm 0.0067$ \\
& 60 & $6.4 \pm 0.05$ & Homogenous & $14.01 \pm 0.067$ & $98.09 \pm 0.044$ \\
$45 \pm 2{ }^{\circ} \mathrm{C}$ & 90 & $5.8 \pm 0.90$ & Homogenous & $11.79 \pm 0.032$ & $95.67 \pm 0.071$ \\
& 30 & $6.4 \pm 0.067$ & Homogenous & $14.01 \pm 0.54$ & $93.55 \pm 0.0230$ \\
& 60 & $6.0 \pm 0.67$ & Homogenous & $13.87 \pm 0.032$ & $87.56 \pm 0.0032$ \\
& 90 & $5.6 \pm 0.32$ & Homogenous & $13.32 \pm 0.078$ & $85.53 \pm 0.0012$ \\
\hline
\end{tabular}

[Values are mean $\pm \mathrm{SD}(n=3)]$

\section{CONCLUSION}

In this study, LBV of imiquimod using PL 90G, ethanol, lactic acid and span 80 in carbopol gel base were successfully prepared and evaluated for their efficacy as treatment option for genital warts. In vitro/in vivo skin permeation/retention studies indicated superiority of these vesicles might be due to reservoir of imiquimod solubilized in lactic acid, and adaptability in lipid bilayers with the help of span 80. Bilayer fluidity evaluation using FTIR data indicated that this formulation enhanced permeation of imiquimod mainly by fluidization and extraction of lipid bilayer. Histological examination confirmed the biocompatibility of gel formulation by showing no inflammation in dorsal skin. No erythema and oedema was observed after topical administration of drug-loaded LBV gel on rat skin. These findings demonstrated that topical administration of low dose Imiquimod loaded LBV gel might become a promising target treatment for genital warts

\section{ACKNOWLEDGEMENT}

Author wishes to thank staff and colleagues of Hindu College of Pharmacy, Sonepat and Apeejay Stya University, Gurgaon for their support and kind suggestions.

\section{CONFLICT OF INTERESTS}

Authors have declared no conflict of interest

\section{REFERENCES}

1. Sexually transmitted diseases treatment guidelines. Centres for disease control and prevention. MMWR Recomm Rep 2002;51:1-78

2. Karnes JB, Usatine RP. Management of external genital warts. Am Fam Physician 2014;90:312-8.

3. Karthigeyan K. Cervical cancer in India and HPV vaccination. Indian J Med Paediatr Oncol 2012;33:7-12.

4. Cox JT, Petry KU, Rylander E, Roy M. Using imiquimod for genital warts in female patients. J Womens Health 2004;13:265-71.

5. Jubin Ryu F, Clarissa Yang A. Review of topical imiquimod in the management of basal cell carcinoma, actinic keratoses, and other skin lesions. Clin Med Ther 2009;1:1557-75.

6. Bhowmik D, Gopinath H, Kumar BP, Duraivel S, Kumar KPS. Recent advances in novel topical drug delivery system. Text Prax Int 2012;1:12-31.

7. Date AA, Naik B, Nagarsenker MS. Novel drug delivery systems: potential in improving topical delivery of antiacne agents. Skin Pharmacol Physiol 2006;19:2-16. 
8. Fox CB, Sivananthan SJ, Duthie MS, Vergara J, Guderian JA, Moon E, et al. A nanoliposome delivery system to synergistically trigger TLR4 AND TLR7. J Nanobiotechnol 2014;12:1-18.

9. Touitou E. Composition of applying active substance to or through the skin. US Patent: 1996. p. 5, 540, 934.

10. Kaur LP, Guleri TK. Topical gel: a recent approach for novel drug delivery. Asian J Biomed Pharm Sci 2013;3:1-5.

11. Shinde M, Gharge V, Gurjar M, Shah M. Effect of penetration enhancer on the in vitro ex vivo permeation of diclofenac gel. Asian J Pharm Clin Res 2014;7:255-9.

12. Akiladevi D, Basak S. Ethosomes-a noninvasive approach for transdermal drug delivery. Int J Curr Pharm Res 2010;2:1-4.

13. Touitou E, Dayan N, Bergelson L, Godin B, Eliaz M. Ethosomesnovel vesicular carriers for enhanced delivery: characterization and skin penetration properties. J Controlled Release 2000;65:403-18.

14. Shen LN, Zhang YT, Wang Q, Xu L, Feng NP. Enhanced in vitro and in vivo skin deposition of apigenin delivered using ethosomes. Int J Pharm 2014;460:280-8.

15. Sheer A, Chauhan M. Ethosomes as vesicular carrier for enhanced transdermal delivery of ketoconazole-formulation and evaluation. J Pharm Cosmetol 2011;1:1-14.

16. Rao $\mathrm{Y}$, Zheng $\mathrm{F}$, Zhang $\mathrm{X}$, Gao J, Liang W. In vitro percutaneous permeation and skin accumulation of finasteride using vesicular ethosomal carrier. AAPS Pharm SciTech 2008;9:860-5.

17. Wavikar P, Vavia P. Nanolipidgel for enhanced skin deposition and improved antifungal activity. AAPS PharmSciTech 2013;14:222-33.

18. Pawar DP, Shamkuwar PB. Formulation and evaluation of herbal gel containing lantana camara leaves extract. Asian J Pharm Clin Res 2013;6:122-4.

19. Meghana G, Narayana Reddy Karri VVS, Talluri SV, Gunda R, Chennareddy SR, Ganesh GNK. Formulation and evaluation of tolnaftate loaded topical liposomal gel for effective skin drug delivery to treat fungal diseases. J Chem Pharm Res 2014;6:856-66.

20. Patel TN, Patel MM. Preparation and evaluation of imiquimod loaded solid lipid nanoparticles based topical gel formulation: part-1. J Biochem Pharmacol Res 2013;2:37-46.

21. Jain S, Tiwary AK, Sapra B, Jain NK. Formulation and evaluation of ethosomes for transdermal delivery of lamivudine. AAPS PharmSciTech 2007;8:111-9.

22. De PD, Martins CA, Entley M, Vitoria LB. Development and validation of HPLC method for imiquimod determination in skin penetration studies. Biomed Chromatogr 2008;22:1416-23.

23. Jain S, Diwan A, Sardana S. Development and validation of UV spectroscopy and RP-HPLC methods for estimation of imiquimod Int J Pharm Sci Rev Res 2015;35:16-21.
24. Ma M, Wang J, Fang G, Lei M, Tan F, Li N. Development of nanovesicular systems for dermal Imiquimod delivery: physicochemical characterization and in vitro/in vivo evaluation. J Mater Sci Maters Med 2015;26:192.

25. Gaur PK, Purohit S, Mishra S. Development of aceclofenac nanovesicular system using biomaterial for transdermal delivery: physical characterization, ex vivo, in vivo and antiinflammatory studies. J Biomater Sci Polym Ed 2013;24:2126-41.

26. Panchaxari DM, Pampana S, Pal T, Devabh Maheshwari RGS, Tekade RK, Sharma PA, et al. Ethosomes and ultradeformable liposomes for transdermal delivery of clotrimazole: a comparative assessment. Saudi Pharm J 2012;20:161-70.

27. Aktuni B, Aravapalli AK. Design and characterization of diclofenac diethylamine transdermal patch using silicon and acrylic adhesives combination. DARU 2013;21:1-14.

28. Gupta V, Dhote V, Paul VN, Trivedi P. Development of novel topical drug delivery system containing cisplatin and imiquimod for dual therapy in cutaneous epithelial malignancy. J Liposome Res 2014;24:50-162.

29. Dubey V, Mishra D, Nahar M, Jain V, Jain NK. Enhanced transdermal delivery of an anti-HIV agent via ethanolic liposomes. Nanomed: Nanotechnol Biol Med 2010;6:590-6.

30. Elsayed MA, Abdallah YO, Naggar FV, Khalafallah NM. Lipid vesicles for skin delivery of drugs: reviewing three decades of research. Int J Pharm 2006;332:1-16.

31. Zhang JP, Wei YH, Zhou Y, Li YQ, Wu XA. Ethosomes, binary ethosomes and transfersomes of terbinafine hydrochloride: a comparative study. Arch Pharm Res 2012;35:109-17.

32. Guo F, Wang JP, Ma M, Tan FP, Li N. Skin targeted lipid vesicles as novel nano-carrier of ketoconazole: characterization, in vitro and in vivo evaluation. J Mater Sci 2015;26:175.

33. Rawat AS, Kumar MS, Khurana B, Mahadevan N. Proniosome gel. Int J Res Ayurveda Pharm 2013;3:3-10.

34. Akhtar N, Pathak K. Cavamax W7 composite ethosomal gel of clotrimazole for improved topical delivery: development and comparison with ethosomal gel. AAPS PharmSciTech 2011;13:344-55.

35. Guiling L, Yating F, Chao F, Xinru L, Xiaoning W, Mei L, et al. Tacrolimus-loaded ethosomes: physicochemical characterization and in vivo evaluation. Eur J Pharm Biopharm 2012;82:49-57.

36. Shiny A, Toomu MC, Dhurke RK. Enhanced dermal delivery of nadifloxacin using liposomes. Int J Appl Pharm 2016;8:53-9.

\section{How to cite this article}

- $\quad$ Saroj Jain, Anupama Diwan, Satish Sardana. Use of lactic acid and span 80 in the formulation of lipid based imiquimod vesicles for genital warts. Int J Pharm Pharm Sci 2017;9(2):292-301. 